\title{
Clone, expression and plasminogen binding property of three fructose-1,6-bisphosphate aldolases from Clonorchis sinensis
}

\author{
Li, S. ${ }^{1,2,3 \#}$, Lu, B.P. ${ }^{1 \#}$, Feng, J. ${ }^{4}$, Zhou, J.J. ${ }^{5}$, Xie, Z.Z. ${ }^{6}$, Liang, C. ${ }^{2,3}$, Li, X.R. ${ }^{2,3}$, Huang, Y..$^{2,3}$ and Yu, X.B. ${ }^{2,3^{*}}$ \\ ${ }^{1}$ School of Basic Medicine, Henan University of Chinese Medicine, Zhengzhou 450046, China \\ ${ }^{2}$ Department of Parasitology, Zhongshan School of Medicine, Sun Yat-sen University, Guangzhou 510080, \\ China \\ ${ }^{3}$ Key Laboratory for Tropical Diseases Control of Ministry of Education, Sun Yat-sen University, \\ Guangzhou 510080, China \\ ${ }^{4}$ Zhengzhou YIHE Hospital Affiliated to Henan University, Zhengzhou 450047, China \\ ${ }^{5}$ Zhengzhou Key Laboratory for Children's Infection and Immunity, Children's Hospital Affiliated to Zhengzhou \\ University, Zhengzhou 450047, China \\ ${ }^{6}$ Department of Clinical Laboratory, Third Affiliated Hospital of Sun Yat-sen University, Guangzhou 510630, \\ China \\ \#These authors contributed equally to this work. \\ *Corresponding author e-mail: yuxinbingteam@163.com \\ Received 19 January 2020; received in revised form 5 September 2020; accepted 9 September 2020
}

\begin{abstract}
Fructose-1,6-bisphosphate aldolase (FbA), a well characterized glycometabolism enzyme, has been found to participate in other important processes besides the classic catalysis. To understand the important functions of three fructose-1,6-bisphosphate aldolases from Clonorchis sinensis (CsFbAs, CsFbA-1/2/3) in host-parasite interplay, the open reading frames of $C s \mathrm{FbAs}$ were cloned into pET30a (+) vector and the resulting recombinant plasmids were transformed into Escherichia coli BL21 (DE3) for expression of the proteins. Purified recombinant $C s \mathrm{FbAs}$ proteins ( $\mathrm{r} C s \mathrm{FbAs}$ ) were approximately $45.0 \mathrm{kDa}$ on $12 \%$ SDS-PAGE and could be probed with each rat anti-rCsFbAs sera by western blotting analysis. ELISA and ligand blot overlay indicated that $\mathrm{rCsFbAs}$ of $45.0 \mathrm{kDa}$ as well as native $C s \mathrm{FbAs}$ of $39.5 \mathrm{kDa}$ from total worm extracts and excretory-secretory products of Clonorchis sinensis (CsESPs) could bind to human plasminogen, and the binding could be efficiently inhibited by lysine analog $\varepsilon$-aminocaproic acid. Our results suggested that as both the components of CsESPs and the plasminogen binding proteins, three $C s \mathrm{FbAs}$ might be involved in preventing the formation of the blood clot so that Clonorchis sinensis could acquire enough nutrients from host tissue for their successful survival and colonization in the host. Our work will provide us with new information about the biological function of three $C s F b A s$ and their roles in hostparasite interplay.
\end{abstract}

\section{INTRODUCTION}

Clonorchiasis, closely related to cholangiocarcinoma and hepatocellular carcinoma, is a major public health problem in globally, especially some Asian countries such as China (including Hong Kong and Taiwan), Japan, Korea, and Vietnam (Li et al., 2020). Clonorchis sinensis (C. sinensis), the causative agent of clonorchiasis, has resulted in a serious socioeconomic burden in epidemic regions. Approximately 35 million people were infected with $C$. sinensis worldwide, including 15 million people in China (Lun et al., 2005). The molecular mechanism involved in clonorchiasis is very complex and incompletely clear. It is generally believed that excretory-secretory products (ESPs), continuously released or exfoliated proteins mixture from C. sinensis, 
are toxic to and interact with host's bile epithelium (Pak et al., 2009). Long-term stimulus from these molecules can provoke DNA mutation and chronic pathologic changes such as inflammation, epithelial hyperplasia, goblet cell metaplasia, periductal fibrosis, and biliary intraepithelial neoplasia (Sripa et al., 2007). Since ESPs of C. sinensis (CsESPs) play a crucial role in host-parasite interaction, studies on them will be an essential step to further exploring the pathogenesis of clonorchiasis. Some proteins from $C s$ ESPs have been proven to participate in nutrients intake and energy metabolism, detoxification of bile components, and immune evasion (Wang et al., 2011a; Huang et al., 2012; Lei et al., 2013; Liang et al., 2013). However, the biological function of most proteins from $C s$ ESPs remains obscure to date.

Fructose-1,6-bisphosphate aldolase (FbA) is a ubiquitous and abundant glycometabolism enzyme that reversibly catalyzes D-fructose-1,6-bisphosphate to yield D-glyceraldehyde-3-phosphate and dihydroxyacetone phosphate (Maurady et al., 2002). In our previous study, three FbA isozymes of $C$. sinensis (CsFbAs, CsFbA-1/ 2/3) were identified as the components of $C s \mathrm{ESPs}$, and two of the recombinant $C s \mathrm{FbAs}$ (rCsFbA-2 and $\mathrm{rCsFbA}-3$ ) showed catalytic activities (Li et al., 2014). Recently, emerging evidences have demonstrated that some ancient metabolic enzyme, such as glyceraldehyde-3-phosphate dehydrogenase, triosephosphate isomerase, and glycogen synthase kinases, can also participate in other important processes besides the classic catalytic activities, termed "moonlighting functions" (Nagini et al., 2018; Sirover, 2018; Malhotra et al., 2019; Rodriguez-Bolanos \& Perez-Montfort, 2019). The "moonlighting functions" of metabolic enzymes are found throughout the archaea, bacteria, fungi, viruses, parasites, plants, insects, reptiles, fishes, birds, and even mammals. These metabolic enzymes play important roles in signal transduction (Kim et al., 2002; Ishida et al., 2005), vesicle trafficking and redistribution (Lu et al., 2001; Lu et al., 2004;
Lu et al., 2007; Merkulova et al., 2011), cell mobility (Kusakabe et al., 1997; Schindler et al., 2001; Tochio et al., 2010), as well as adhesion and invasion of the pathogen (Jewett \& Sibley, 2003; Starnes et al., 2009; Tunio et $a l ., 2010)$. It has been reported that metabolic enzymes of the pathogen present the "moonlighting functions" mostly relate to its virulence activity of the infection (FrancoSerrano et al., 2018). Therefore, understanding the "moonlighting functions" of $C s F b A s$ will help us to find the molecular mechanisms of C. sinensis invading and infecting the host.

In the present study, three $C s \mathrm{FbAs}$ were overexpressed in Escherichia coli (E. coli) BL21 (DE3) and their plasminogen binding properties were investigated. Our work will enrich the knowledge about the biological functions of $C s \mathrm{FbAs}$ in host-parasite interplay and facilitate the explanation of the pathogenesis of clonorchiasis.

\section{MATERIALS AND METHODS}

\section{Animals and parasites}

Six-week-old male Sprague-Dawley (SD) rats were purchased from the animal center of Sun Yat-sen University. All animal experiments were approved by the Animal Care and Use Committee of Sun Yat-sen University (permit number SYXK (Guangdong) 20120081), and performed in accordance with Guangdong Provincial Laboratory Animal Administration Measures and the Laboratory Animal Administration Regulations issued by Ministry of Science and Technology of the People's Republic of China. Metacercariae of $C$. sinensis were isolated from experimentally infected Pseudorasbora parva in our ecologic pool (Liang et al., 2009). The fish was digested with pepsin- $\mathrm{HCl}(\mathrm{pH} 2.0)$ for $2 \mathrm{~h}$ at $37^{\circ} \mathrm{C}$, and then filtered through the sieve mesh. The living metacercariae were washed several times with sterile phosphatebuffered saline (PBS) and collected under a light microscope. Adult worms were freshly collected from the bile ducts of SD rats infected with metacercariae for 8 weeks. After washing 3 times with sterile PBS, adult 
worms were incubated in Sample Protecter (TaKaRa, Japan) and frozen in $-80^{\circ} \mathrm{C}$ for RNA and protein extraction.

\section{Acquirement of recombinant CsFbAs proteins ( $\mathrm{rCsFbAs)}$ and antisera}

The complete encoding sequences of $C s \mathrm{FbA}-1, C s \mathrm{FbA}-2$, and $C s \mathrm{FbA}-3$ (GenBank accession numbers: DF143094.1, DF143896.1, and DF143689.1) were obtained as described (Li et al., 2014). Total RNA from C. sinensis adults was extracted with TRIZOL reagent (Invitrogen, USA) and the cDNA was synthesized by using the Reverse Transcriptase XL (TaKaRa, Japan). The open reading frames (ORF) of three $C s \mathrm{FbAs}$ were amplified from cDNA of adult worms by using specific primers containing restriction enzyme sites as described (Li et al., 2014). The purified PCR products were cloned into the $6 \times$ His tagged pET30a (+) vector (Novagen, USA). The resulting recombinant plasmids were identified by agarose gel electrophoresis and DNA sequencing, and then transformed into $E$. coli BL21 (DE3) (Promega, USA) for expression. The expression of recombinant proteins was induced by adding $0.5 \mathrm{mM}$ isopropyl- $\beta$-Dthiogalactopyranoside (IPTG) at $37^{\circ} \mathrm{C}$ for $4 \mathrm{~h}$ in Luria-Bertani medium. The induced cells were collected by centrifugation at $8,000 \mathrm{~g}$ for $15 \mathrm{~min}$ at $4^{\circ} \mathrm{C}$, suspended in lysis buffer (0.5 M NaCl, $20 \mathrm{mM}$ Tris-HCl, $5 \mathrm{mM}$ imidazole; $\mathrm{pH}$ 8.0), and then sonicated for $20 \mathrm{~min}$ in an ice bath. The supernatants were obtained by centrifugation at $12,000 \mathrm{~g}$ for $15 \mathrm{~min}$ at $4^{\circ} \mathrm{C}$ and purified by His Bind Purification Kit (Novagen, USA). The purified proteins were collected through gradient elution with 5-300 $\mathrm{mM}$ imidazole buffer and evaluated by $12 \%$ sodium dodecyl sulfate-polyacrylamide gel electrophoresis (SDS-PAGE). Concentration of three purified $\mathrm{rCsFbAs}$ was measured by BCA Assay Kit (Novagen, USA).

To obtain antisera, each rat was subcutaneously immunized with $200 \mu \mathrm{g} r C s F b A-$ 1, rCsFbA-2, or $\mathrm{rCsFbA-3} \mathrm{emulsified} \mathrm{with}$ equal volume of complete Freund's adjuvant (Sigma, USA). Two booster immunizations were performed at 2-week intervals with $100 \mu \mathrm{g}$ rCsFbA-1, rCsFbA-2, or $\mathrm{rCsFbA}-3$ emulsified with equal volume of incomplete Freund's adjuvant (Sigma, USA). Anti$\mathrm{r} C s \mathrm{FbAs}$ sera were obtained 2 weeks after the last immunization. Sera from unimmunized rats were also obtained and used as the control. Sera were stored at $-80^{\circ} \mathrm{C}$ until use. Antibody titers were determined by enzyme-linked immunosorbent assay (ELISA).

\section{Western blotting analysis of $\mathrm{rCsFbAs}$ and native $C s$ FbAs}

Worm extracts (WEs) and CsESPs were respectively prepared according to the previous method and their concentrations were measured by BCA Assay Kit (Novagen, USA) (Wang et al., 2011a). Purified rCsFbAs, WEs, and CsESPs were subjected to $12 \%$ SDS-PAGE and electrotransferred onto polyvinylidene difluoride (PVDF) membranes (Millipore, USA). The membranes were blocked with $5 \%$ skim milk in PBS containing $0.05 \%$ Tween 20 (PBS-T) at room temperature (RT) for $3 \mathrm{~h}$, and then incubated with rat anti$\mathrm{r} C s \mathrm{FbAs}$ sera or unimmunized sera $(1: 1,000$ dilutions in PBS-T with 5\% BSA) at $4^{\circ} \mathrm{C}$ overnight. After washing thoroughly, the membranes were incubated with horseradish peroxidase (HRP)-conjugated goat anti-rat IgG antibody (ProteinTech, USA; 1:10,000 dilutions) at RT for $1 \mathrm{~h}$. Blots on the membranes were detected using an enhanced chemiluminescence (ECL) method (Millipore, USA).

\section{Plasminogen binding analysis of rCsFbAs and native $C \boldsymbol{s F b A s}$}

Plasminogen binding properties of $\mathrm{rCsFbAs}$ were determined by ELISA as described (de la Paz Santangelo et al., 2011). Wells coated with $2 \mu \mathrm{g}$ of purified $\mathrm{rCsFbA}-1, \mathrm{rCsFbA}-2$, $\mathrm{rCsFbA}-3$, fibrinogen (Calbiochem, Germany; positive control), or BSA (negative control) were respectively incubated with $50 \mu \mathrm{M}$ human plasminogen (Athen, USA) in PBS-T with $1 \% \mathrm{BSA}$ at $37^{\circ} \mathrm{C}$ for $2 \mathrm{~h}$ in the presence of $0,50,100$, and $200 \mathrm{mM}$-aminocaproic acid ( $\varepsilon$-ACA). In addition, wells coated with $2 \mu \mathrm{g}$ of $\mathrm{rCs} \mathrm{FbA}-1, \mathrm{rCsFbA}-2$, rCsFbA-3, or BSA were incubated with $0-10.24 \mu \mathrm{M}$ plasminogen, respectively. Rabbit anti-human plasminogen 
polyclonal antibody (ProteinTech, USA; 1:5,000 dilutions) and HRP-conjugated goat anti-rabbit IgG antibody (1:20,000 dilutions) were used as the primary antibody and the secondary antibody, respectively. The reactions were visualized by TMB substrate solution (BD Biosciences) at OD $450 \mathrm{~nm}$.

Ligand blot overlay assay was used to identify the binding property of $\mathrm{rCsFbAs}$ and native $C s \mathrm{FbAs}$ to human plasminogen according to the previous method (Lin $e t$ $a l$., 2011). Purified $\mathrm{rCsFbA}-1, \mathrm{rCsFbA}-2$, or rCsFbA-3, as well as WEs and CsESPs were subjected to $12 \%$ SDS-PAGE and electrotransferred onto PVDF membranes. The membranes were blocked with PBS-T containing 5\% skim milk at RT for $3 \mathrm{~h}$, and then incubated with $50 \mu \mathrm{M}$ human plasminogen in the presence or absence of $200 \mathrm{mM} \varepsilon$-ACA at $4{ }^{\circ} \mathrm{C}$ overnight. After washing process, the membranes were incubated with rabbit anti-human plasminogen polyclonal antibody (1:5,000 dilutions) at $4{ }^{\circ} \mathrm{C}$ overnight, and subsequently incubated with HRP-conjugated goat antirabbit IgG antibody (1:10,000 dilutions) at $\mathrm{RT}$ for $1 \mathrm{~h}$. The blots on the membranes were detected by the ECL method.

\section{Statistical analysis}

Statistical analysis was performed using SPSS software 19.0. Data were represented as mean \pm SD from at least 3 separate experiments.

\section{RESULTS}

Agarose gel electrophoresis identification of recombinant pET-30a (+)CsFbAs plasmids

Recombinant three pET-30a (+)-CsFbAs plasmids were respectively digested by restriction enzymes and identified by agarose gel electrophoresis, showing the bands of approximately 1,000 base pairs (bp) and $5,000 \mathrm{bp}$. The bands of 1,000 bp were in accordance with PCR amplification of three CsFbAs ORFs (CsFbA-1, 1089bp; CsFbA-2, 1092bp; CsFbA-3, 1092bp), while the bands of 5,000 bp were in accordance with pET-30a $(+)$ digested by restriction enzymes (Figure 1A-C).

\section{Gradient elution of $\mathrm{rCs}$ FbAs with imidazole buffer}

Imidazole eluent of $\mathrm{rCsFbAs}$ was analyzed by SDS-PAGE for collection of the purified proteins. With the increasing concentration of imidazole, three $\mathrm{rCs} \mathrm{FbAs}$ were gradually purified, showing the bands of approximately $45.0 \mathrm{kDa}$ on $12 \%$ SDS-PAGE. Purified rCsFbA-1 were collected from 150, 200, and $300 \mathrm{mM}$ imidazole eluent, purified $\mathrm{rCsFbA}-2$ were collected from $100 \mathrm{mM}$ and $150 \mathrm{mM}$ imidazole eluent, and purified $\mathrm{r} C s \mathrm{FbA}-3$ were collected from 60,80 , and $100 \mathrm{mM}$ imidazole eluent (Figure 2A-C).

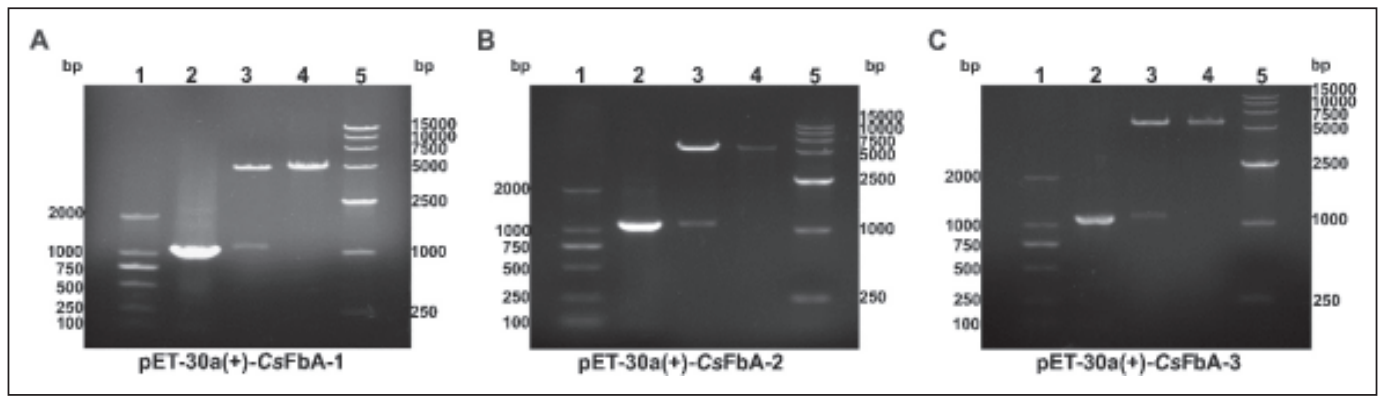

Figure 1. Agarose gel electrophoresis identification of recombinant pET-30a (+)-CsFbAs plasmids. (A) pET-30a (+)-CsFbA-1. (B) pET-30a (+)-CsFbA-2. (C) pET-30a (+)-CsFbA-3. DL2000 Maker (lane 1), PCR amplification products (lane 2), pET-30a (+)-CsFbA-1, pET-30a (+)-CsFbA-2 or pET-30a (+)-CsFbA-3 digested by restriction enzymes (lane 3), pET-30a (+) digested by restriction enzymes (lane 4), and DL15000 Maker (lane 5). 


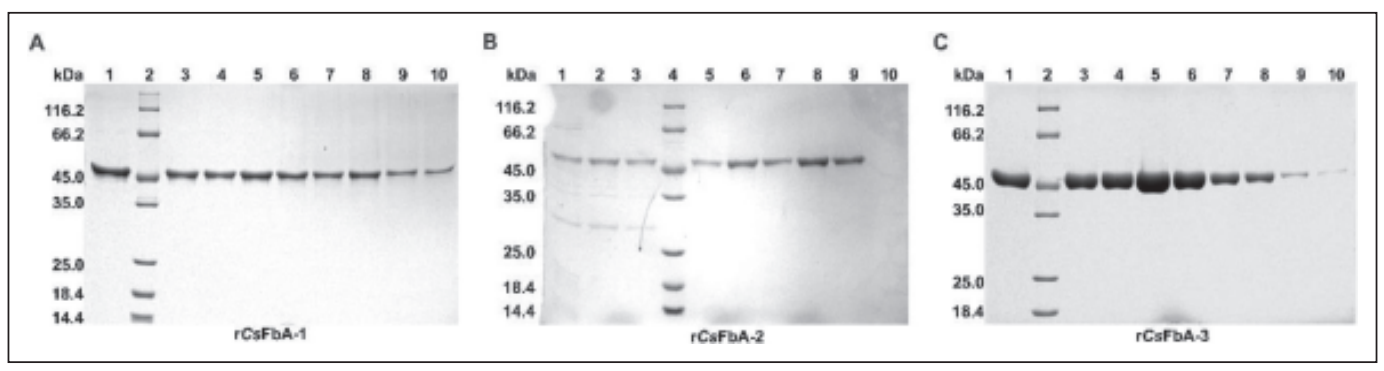

Figure 2. Gradient elution of $\mathrm{r} C s \mathrm{FbAs}$ with imidazole buffer. Imidazole eluent of $\mathrm{r} C s \mathrm{FbAs}$ was analyzed by SDS-PAGE for collection of the purified proteins. (A) rCsFbA-1. Protein MV marker (lane 2), 150 mM imidazole eluent (lane 1, 3 and 4), $200 \mathrm{mM}$ imidazole eluent (lane 5, 6 and 7), $300 \mathrm{mM}$ imidazole eluent (lane 8, 9 and 10). (B) rCsFbA-2. Protein MV marker (lane 4), $80 \mathrm{mM}$ imidazole eluent (lane 1, 2 and 3), $100 \mathrm{mM}$ imidazole eluent (lane 5, 6 and 7), $150 \mathrm{mM}$ imidazole eluent (lane 8, 9 and 10). (C) rCsFbA-3. Protein MV marker (lane 2), $60 \mathrm{mM}$ imidazole eluent (lane 1, 3 and 4), $80 \mathrm{mM}$ imidazole eluent (lane 5, 6 and 7), $100 \mathrm{mM}$ imidazole eluent (lane 8, 9 and 10).

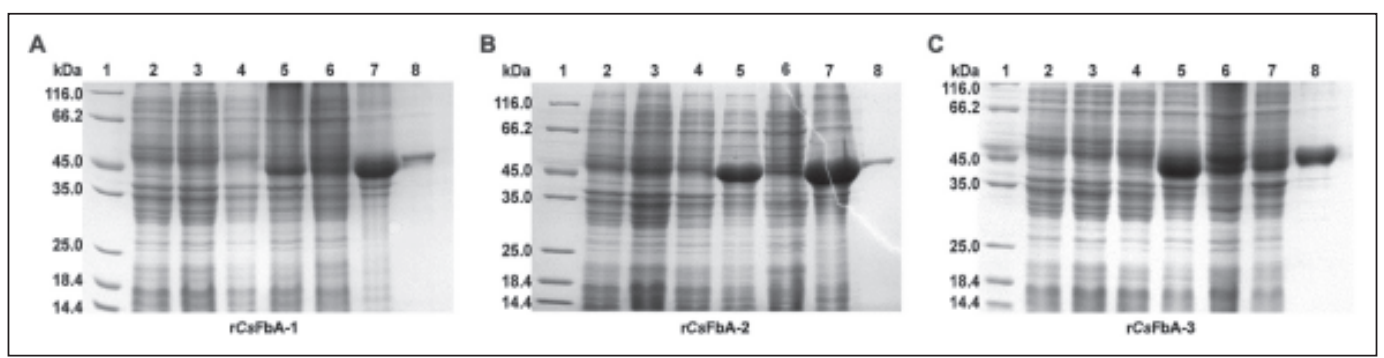

Figure 3. SDS-PAGE identification of rCsFbAs. (A) rCsFbA-1. (B) rCsFbA-2. (C) rCsFbA-3. Protein MV marker (lane 1), lysate of $E$. coli with pET-30a (+) before and after induction (lane 2 and 3), lysate of E. coli with pET-30a (+)-CsFbA-1, pET-30a (+)-CsFbA-2 or pET-30a (+)-CsFbA-3 before and after induction (lane 4 and 5), supernatant and precipitation of induced pET-30a (+)-CsFbA-1, pET-30a (+)CsFbA-2 or pET-30a (+)-CsFbA-3 transformants (lane 6 and 7), and purified rCsFbA-1, rCsFbA-2 or $\mathrm{rCsFbA}-3$ (lane 8).

\section{SDS-PAGE identification of $\boldsymbol{C s F b A s}$}

Purified proteins of three $\mathrm{rCsFbAs}$ with $6 \times$ His-tag were approximately $45.0 \mathrm{kDa}$ confirmed by $12 \%$ SDS-PAGE, which were consistent with their theoretical molecular weight (MW; rCsFbA-1, $45.0 \mathrm{kDa}$; $\mathrm{rCsFbA-2}$, $45.2 \mathrm{kDa} ; \mathrm{rCsFbA}-3,45.2 \mathrm{kDa}$ ), including the extra amino acids from vectors (Figure 3A-C).

\section{ELISA assay of IgG titers from anti- CsFbAs sera}

After rats immunized with $\mathrm{rCsFbAs,} \mathrm{IgG}$ antibody titers were assayed by ELISA. IgG titers in anti-rCsFbA-1, anti-rCsFbA-2, and anti-rCsFbA-3 sera were respectively
1:102400, 1:204800, and 1:204800, indicating that high levels of IgG antibodies were obtained after rats immunized with $\mathrm{rCsFbAs}$ (Figure 4A-C).

\section{Western blotting analysis of rCsFbAs and native $C s F b A s$}

Purified $\mathrm{rCsFbAs}$ could be probed with each rat anti-rCsFbAs sera at the band of approximately $45.0 \mathrm{kDa}$, while WEs and $C s$ ESPs could be probed with each rat anti$\mathrm{rCsFbAs}$ sera at the bands of approximately $39.5 \mathrm{kDa}$ (MW of native CsFbAs). However, purified rCsFbAs, WEs, and CsESPs could not be probed with unimmunized sera (Figure 4D-F). 


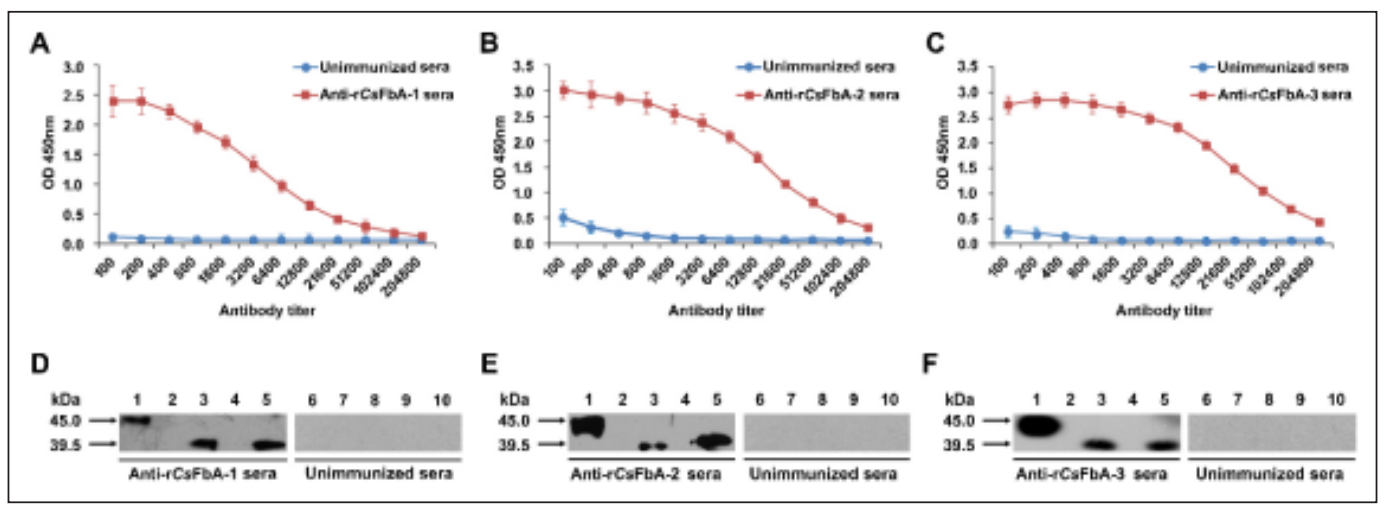

Figure 4. Western blotting analysis of $\mathrm{r} C s \mathrm{FbAs}$ and native $C s \mathrm{FbAs}$. IgG antibody titer in anti-rCsFbA-1 sera (A), anti-rCsFbA-2 sera (B) and anti-rCsFbA-3 sera (C). $\mathrm{P} / \mathrm{N} \geq 2.1$ was considered as an effective antibody titer (P, OD 450nm of anti-rCsFbAs sera; N, OD 450nm of unimmunized sera). Western blotting analysis of $\mathrm{rCsFbA}-1$ (D), $\mathrm{rCsFbA}-2(\mathrm{E}), \mathrm{r} C s \mathrm{FbA}-3(\mathrm{~F})$, and native $C s \mathrm{FbAs}$ from WEs or CsESPs. Purified rCsFbA-1, rCsFbA-2, or rCsFbA-3 (lane 1 and 6), $1 \times$ loading buffer (lane 2 and 7), WEs (lane 3 and 8), 1× loading buffer (lane 4 and 9) and CsESPs (lane 5 and 10).

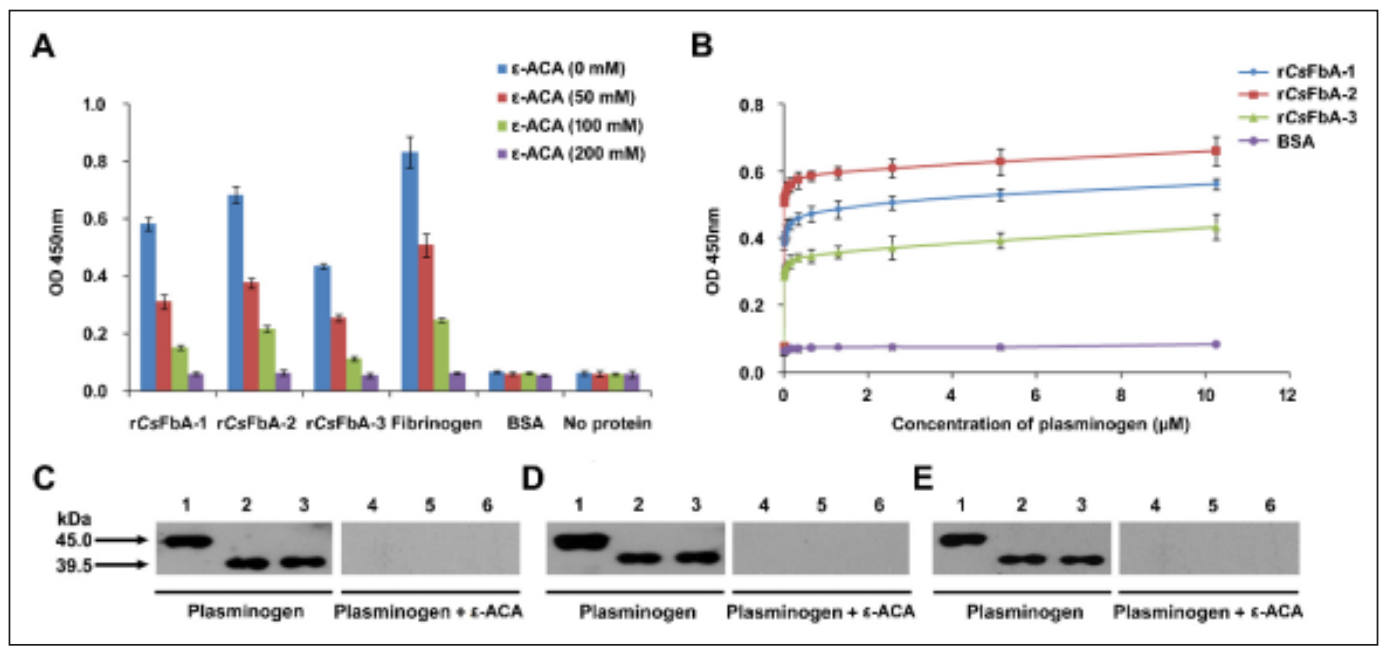

Figure 5. Plasminogen binding property of $\mathrm{r} C s \mathrm{FbAs}$ and native $C s \mathrm{FbAs}$. (A) Plasminogen binding property of $\mathrm{rCsFbAs}$ assayed by ELISA. Wells coated with $2 \mu \mathrm{g}$ of $\mathrm{r} C s \mathrm{FbA}-1, \mathrm{rCsFbA}-2, \mathrm{r} C s \mathrm{FbA}-3$, fibrinogen (positive control), or BSA (negative control) was incubated with $50 \mu \mathrm{M}$ plasminogen in the presence of $0,50,100$ and $200 \mathrm{mM} \varepsilon$-ACA. (B) Dose-dependent binding property of $\mathrm{r} C s \mathrm{FbAs}$ to plasminogen assayed by ELISA. Wells coated with $2 \mu \mathrm{g}$ of $\mathrm{r} C s \mathrm{FbA}-1, \mathrm{r} C s \mathrm{FbA}-2, \mathrm{r} C s \mathrm{FbA}-3$, or BSA (control) was incubated with $0-10.24 \mu \mathrm{M}$ plasminogen. Plasminogen binding property of $\mathrm{rCsFb}-1$ (C), rCsFbA-2 (D), rCsFbA-3 (E), and native $C s F b A s$ from WEs or CsESPs assayed by ligand blot overlay. Purified rCsFbA-1, rCsFbA-2, or rCsFbA-3 (lane 1 and 4), WEs (lane 2 and 5), and CsESPs (lane 3 and 6).

Plasminogen binding property of rCsFbAs and native $C s$ FbAs

ELISA assay showed that the OD 450nm values of $\mathrm{r} C s \mathrm{FbA}-1, \mathrm{r} C s \mathrm{FbA}-2, \mathrm{rCsFbA}-3$, and fibrinogen were significantly higher than that of BSA when they were incubated with
$50 \mu \mathrm{M}$ human plasminogen in the absence of $\varepsilon$-ACA. The binding of three $\mathrm{rCsFbAs}$ or fibrinogen to human plasminogen could be inhibited by 50, 100, and $200 \mathrm{mM}$ lysine analogue $\varepsilon$-ACA (Figure 5A). In addition, the binding of three $\mathrm{rCsFbAs}$ to plasminogen 
exhibited a dose-dependent manner. With the increasing concentration of plasminogen $(0-10.24 \mu \mathrm{M})$, the binding affinity of three $\mathrm{rCsFbAs}$ was gradually strengthened. Apparently, rCsFbA-2 protein showed the strongest plasminogen binding affinity, while $\mathrm{r} C s \mathrm{FbA}-3$ protein showed the weakest plasminogen binding affinity (Figure 5B).

Ligand blot overlay assay showed that three $\mathrm{rCsFbAs}$ incubated with human plasminogen could be blotted with rabbit anti-human plasminogen polyclonal antibody at the bands of approximately $45.0 \mathrm{kDa}$ (MV of $\mathrm{rCsFbAs}$ ) in the absence of $\varepsilon$-ACA. WEs or $C s$ ESPs incubated with human plasminogen could also be blotted with rabbit anti-human plasminogen polyclonal antibody at the bands of approximately $39.5 \mathrm{kDa}$ (MV of native $C s \mathrm{FbAs}$ ) in the absence of $\varepsilon$-ACA. However, neither $\mathrm{rCsFbAs}$ nor WEs or CsESPs incubated with human plasminogen could be blotted with rabbit anti-human plasminogen polyclonal antibody in the presence of $200 \mathrm{mM} \varepsilon$-ACA (Figure 5C-E).

\section{DISCUSSION}

FbA is a well-known glycometabolism enzyme responsible for energy production and metabolic processes in almost all organisms. Recently, it has been found to exert unusual "moonlighting functions" besides its glycometabolism activity (Shams et al., 2014). To assess possible "moonlighting functions" of $\mathrm{CsFbAs}$, three isozymes were overexpressed in $E$. coli BL21 (DE3) and the purified proteins with expected MV of $45.0 \mathrm{kDa}$ were obtained (Figure 1-3). FbA has been commonly identified as a molecule of tegument and ESPs from other trematodes including Opisthorchis viverrini (Mulvenna et al., 2010), Fasciola hepatica (Jefferies et al., 2001; Wilson et al., 2011), Schistosoma japonicum (Liu et al., 2009), and Schistosoma bovis (Perez-Sanchez et al., 2006). In the present study, rCsFbAs of $45.0 \mathrm{kDa}$ and the native $C s \mathrm{FbAs}$ of $39.5 \mathrm{kDa}$ from ESPs and WEs could be blotted with anti-CsFbAs sera by western blotting analysis, indicating that $C s \mathrm{FbAs}$ were the components of $C s \mathrm{ESPs}$
(Figure 4D-F). This result was consistent with the previous studies (Wang et al., 2011b; Li et al., 2014), implying that as the components of $C s \mathrm{ESPs}, C s \mathrm{FbAs}$ could directly react with the bile duct of host and might play a moonlighting role in host-parasite interaction.

$\mathrm{FbA}$ has been proven to be a human plasminogen binding and activating protein in parasite (Ramajo-Hernandez et al., 2007; Lorenzatto et $a l .$, 2012) and non-parasite (Crowe et al., 2003; Shams et al., 2016). The phenomenon of human plasminogen binding is commonly found in bacteria (Boyle \& Lottenberg, 1997), fungi (Crowe et al., 2003), protozoa (Mundodi et al., 2008; GomezArreaza et al., 2011; Figuera et al., 2013), and helminth infection (Jolodar et al., 2003; Bernal et al., 2004; Ramajo-Hernandez et al., 2007; de la Torre-Escudero et al., 2010). These pathogens can utilize host plasminogen to generate proteolytic enzyme plasmin, which is responsible for degradation of the fibrin and the extracellular matrix, activation of the procollagenase, and release of the peptide for nutrition (Lahteenmaki et al., 2001; Gomez-Arreaza et al., 2011). In the present study, both $\mathrm{rCsFbAs}$ as well as native $C s \mathrm{FbAs}$ from ESPs and WEs could efficiently bind with human plasminogen by ELISA and ligand blot overlay assay (Figure 5A-E). As the components of $C s \mathrm{ESPs}$, the host plasminogen binding property of three $C s F b A s$ is probably beneficial for $C$. sinensis to invade and infect the host (Avilan $e t$ al., 2011). During $C$. sinensis infection, the feeding and moving worms hook onto the biliary epithelium by their muscular suckers and continuous uptake blood from the small vessels of host for energy supply (Hou, 1955; Rim, 1986). Therefore, as both the components of $C s$ ESPs and the plasminogen binding protein, $C s \mathrm{FbAs}$ might be involved in preventing formation of the blood clot so that the worms can acquire enough nutrients from the small vessels for their survival and colonization in the host. Plasminogenreceptor proteins possess carboxy-terminal lysine residues that may regulate their binding to plasminogen (Plow et al., 1995). Lysine analogue $\varepsilon$-ACA can competitively inhibit plasminogen binding (Crowe et al., 
2003; Gomez-Arreaza et al., 2011; Figuera et al., 2013). In our study, plasminogen binding property of three $\mathrm{rCs} \mathrm{FbAs}$ could be effectively inhibited by lysine analog $\varepsilon$-ACA (Figure 5A and C-E), implying that $C s F b A s$ possibly contain carboxy-terminal lysine residues as the sites for plasminogen binding. Terminal lysine of Neisseria meningitidis FbA replaced by alanine did not completely block the binding and the residual binding was still blocked by lysine analog $\varepsilon$-ACA, suggesting that several lysine residues within $\mathrm{FbA}$ might contribute to the interaction with plasminogen (Shams et al., 2016). Thus, it is speculated that plasminogen binding affinity of $\mathrm{rCsFbAs}$ may depend on the density of terminal lysine sites. Further study will be necessary to identify the precise location of carboxy-terminal lysine residues in the amino acid sequences of three $C s \mathrm{FbAs}$.

In summary, three $C s \mathrm{FbAs}$ was demonstrated to be of plasminogen binding property for the first time, suggesting that they might participate in preventing formation of the blood clot for adult worms to acquire enough nutrients from the host blood. Our results favored the speculation that three $C s \mathrm{FbAs}$ possibly exert "moonlighting functions" besides its catalytic effect as a glycometabolism enzyme, which will help us to getting new information about the biological function of three $C s \mathrm{FbAs}$ and their role in host-parasite interplay. Further studies will focus on the detailed "moonlighting functions" of three CsFbAs participating in the pathophysiological processes of $C$. sinensis infection.

Acknowledgements. This work was funded by the National Key Basic Research and Development Project (973 project, No. 2010CB530000), the National Natural Science Foundation of China (No. 81101270 and No. 81171602), the National Important Sci-Tech Special Projects (2012ZX1000 4220), the Fundamental Research Funds for the Central Universities (No. 3164015), the Scientific and Technological Project of Henan Province (No. 182102310319), the Important Scientific Research Project of Henan Province for Universities (No.
21B310003), and the Doctoral Research Fund of Henan University of Chinese Medicine (No. BSJJ2015-06). The funders had no role in study design, data collection and analysis, decision to publish, or preparation of the manuscript.

\section{Conflict of Interests}

The authors declare that they have no competing interests.

\section{REFERENCES}

Avilan, L., Gualdron-Lopez, M., Quinones, W., Gonzalez-Gonzalez, L., Hannaert, V., Michels, P.A. \& Concepcion, J.L. (2011). Enolase: a key player in the metabolism and a probable virulence factor of trypanosomatid parasites-perspectives for its use as a therapeutic target. Enzyme Research 2011: 932549.

Bernal, D., de la Rubia, J.E., CarrascoAbad, A.M., Toledo, R., Mas-Coma, S. \& Marcilla, A. (2004). Identification of enolase as a plasminogen-binding protein in excretory-secretory products of Fasciola hepatica. FEBS Letters 563: 203-206.

Boyle, M.D. \& Lottenberg, R. (1997). Plasminogen activation by invasive human pathogens. Thrombosis and Haemostasis 77: 1-10.

Crowe, J.D., Sievwright, I.K., Auld, G.C., Moore, N.R., Gow, N.A. \& Booth, N.A. (2003). Candida albicans binds human plasminogen: identification of eight plasminogen-binding proteins. Molecular Microbiology 47: 1637-1651.

de la Paz Santangelo, M., Gest, P.M., Guerin, M.E., Coincon, M., Pham, H., Ryan, G., Puckett, S.E., Spencer, J.S., GonzalezJuarrero, M., Daher, R., Lenaerts, A.J., Schnappinger, D., Therisod, M., Ehrt, S., Sygusch, J. \& Jackson, M. (2011). Glycolytic and non-glycolytic functions of Mycobacterium tuberculosis fructose1,6-bisphosphate aldolase, an essential enzyme produced by replicating and non-replicating bacilli. Journal of Biological Chemistry 286: 40219-40231. 
de la Torre-Escudero, E., Manzano-Roman, R., Perez-Sanchez, R., Siles-Lucas, M. \& Oleaga, A. (2010). Cloning and characterization of a plasminogen-binding surface-associated enolase from Schistosoma bovis. Veterinary Parasitology 173: 76-84.

Figuera, L., Acosta, H., Gomez-Arreaza, A., Davila-Vera, D., Balza-Quintero, A., Quinones, W., Mendoza-Briceno, R.V., Concepcion, J.L. \& Avilan, L. (2013). Plasminogen binding proteins in secreted membrane vesicles of Leishmania mexicana. Molecular and Biochemical Parasitology 187: 14-20.

Franco-Serrano, L., Cedano, J., Perez-Pons, J.A., Mozo-Villarias, A., Pinol, J., Amela, I. \& Querol, E. (2018). A hypothesis explaining why so many pathogen virulence proteins are moonlighting proteins. Pathogens and Disease 76.

Gomez-Arreaza, A., Acosta, H., BarrosAlvarez, X., Concepcion, J.L., Albericio, F. \& Avilan, L. (2011). Leishmania mexicana: LACK (Leishmania homolog of receptors for activated C-kinase) is a plasminogen binding protein. Experimental Parasitology 127: 752-761.

Hou, P.C. (1955). The pathology of Clonorchis sinensis infestation of the liver. The Journal of Pathology and Bacteriology 70: 53-64.

Huang, L., Hu, Y., Huang, Y., Fang, H., Li, R., Hu, D., Li, W., Li, X., Liang, C. \& Yu, X. (2012). Gene/protein expression level, immunolocalization and binding characteristics of fatty acid binding protein from Clonorchis sinensis (CsFABP). Molecular and Cellular Biochemistry 363: 367-376.

Ishida, A., Tada, Y., Nimura, T., Sueyoshi, N., Katoh, T., Takeuchi, M., Fujisawa, H., Taniguchi, T. \& Kameshita, I. (2005). Identification of major $\mathrm{Ca}^{2+} /$ calmodulindependent protein kinase phosphatasebinding proteins in brain: biochemical analysis of the interaction. Archives of Biochemistry and Biophysics 435 : 134-146.
Jefferies, J.R., Campbell, A.M., van Rossum, A.J., Barrett, J. \& Brophy, P.M. (2001). Proteomic analysis of Fasciola hepatica excretory-secretory products. Proteomics 1: 1128-1132.

Jewett, T.J. \& Sibley, L.D. (2003). Aldolase forms a bridge between cell surface adhesins and the actin cytoskeleton in apicomplexan parasites. Molecular Cell 11: 885-894.

Jolodar, A., Fischer, P., Bergmann, S., Buttner, D.W., Hammerschmidt, S. \& Brattig, N.W. (2003). Molecular cloning of an alphaenolase from the human filarial parasite Onchocerca volvulus that binds human plasminogen. Biochimica et Biophysica Acta 1627: 111-120.

Kim, J.H., Lee, S., Lee, T.G., Hirata, M., Suh, P.G. \& Ryu, S.H. (2002). Phospholipase D2 directly interacts with aldolase via its $\mathrm{PH}$ domain. Biochemistry 41: 3414-3421.

Kusakabe, T., Motoki, K. \& Hori, K. (1997). Mode of interactions of human aldolase isozymes with cytoskeletons. Archives of Biochemistry and Biophysics 344: 184-193.

Lahteenmaki, K., Kuusela, P. \& Korhonen, T.K. (2001). Bacterial plasminogen activators and receptors. FEMS Microbiology Reviews 25: 531-552.

Lei, H., Tian, Y., Chen, W., Wang, X., Li, X., Mao, Q., Sun, J., Li, R., Xu, Y., Liang, C., Huang, Y. \& Yu, X. (2013). The biochemical and immunological characterization of two serpins from Clonorchis sinensis. Molecular Biology Reports 40: 3977-3985.

Li, S., Bian, M., Wang, X., Chen, X., Xie, Z., Sun, H., Jia, F., Liang, P., Zhou, C., He, L., Mao, Q., Huang, B., Liang, C., Wu, Z., Li, X., Xu, J., Huang, Y. \& Yu, X. (2014). Molecular and biochemical characterizations of three fructose-1,6bisphosphate aldolases from Clonorchis sinensis. Molecular and Biochemical Parasitology 194: 36-43. 
Li, S., Chen, X., Zhou, J., Xie, Z., Shang, M., He, L., Liang, P., Chen, T., Mao, Q., Liang, C., Li, X., Huang, Y. \& Yu, X. (2020). Amino acids serve as an important energy source for adult flukes of Clonorchis sinensis. PLoS Neglected Tropical Diseases 14: e0008287.

Liang, C., Hu, X.C., Lv, Z.Y., Wu, Z.D., Yu, X.B., Xu, J. \& Zheng, H.Q. (2009). Experimental establishment of life cycle of Clonorchis sinensis. Zhongguo Ji Sheng Chong Xue Yu Ji Sheng Chong Bing Za Zhi 27: 148150.

Liang, P., Sun, J., Huang, Y., Zhang, F., Zhou, J., Hu, Y., Wang, X., Liang, C., Zheng, M., Xu, Y., Mao, Q., Hu, X., Li, X., Xu, J., Lu, G. \& Yu, X. (2013). Biochemical characterization and functional analysis of fructose-1,6-bisphosphatase from Clonorchis sinensis. Molecular Biology Reports 40: 4371-4382.

Lin, D.D., Liu, Y.M., Hu, F., Li, Y.F., Tao, B., Yuan, M., Xie, S.Y., Huang, M.J., Jiang, Q.L., Li, J.Y., Gao, Z.L. \& Wang, J.M. (2011). Evaluation on application of common diagnosis methods for schistosomiasis japonica in endemic areas of China. III. Analysis and evaluation of underestimation of prevalence of Schistosoma japonicum infection by routine Kato-Katz technique. Zhongguo Xue Xi Chong Bing Fang Zhi Za Zhi 23: 642-647.

Liu, F., Cui, S.J., Hu, W., Feng, Z., Wang, Z.Q. \& Han, Z.G. (2009). Excretory/secretory proteome of the adult developmental stage of human blood fluke, Schistosoma japonicum. Molecular \& Cellular Proteomics 8: 1236-1251.

Lorenzatto, K.R., Monteiro, K.M., Paredes, R., Paludo, G.P., da Fonseca, M.M., Galanti, N., Zaha, A. \& Ferreira, H.B. (2012). Fructose-bisphosphate aldolase and enolase from Echinococcus granulosus: genes, expression patterns and protein interactions of two potential moonlighting proteins. Gene 506: 76-84.

Lu, M., Ammar, D., Ives, H., Albrecht, F. \& Gluck, S.L. (2007). Physical interaction between aldolase and vacuolar $\mathrm{H}^{+}$ATPase is essential for the assembly and activity of the proton pump. Journal of Biological Chemistry 282: 24495-24503.
Lu, M., Holliday, L.S., Zhang, L., Dunn, W.A., Jr. \& Gluck, S.L. (2001). Interaction between aldolase and vacuolar $\mathrm{H}^{+}$-ATPase: evidence for direct coupling of glycolysis to the ATP-hydrolyzing proton pump. Journal of Biological Chemistry 276 : 30407-30413.

Lu, M., Sautin, Y.Y., Holliday, L.S. \& Gluck, S.L. (2004). The glycolytic enzyme aldolase mediates assembly, expression, and activity of vacuolar $\mathrm{H}^{+}$-ATPase. Journal of Biological Chemistry 279: 8732-8739.

Lun, Z.R., Gasser, R.B., Lai, D.H., Li, A.X., Zhu, X.Q., Yu, X.B. \& Fang, Y.Y. (2005). Clonorchiasis: a key foodborne zoonosis in China. The Lancet Infectious Diseases 5: $31-41$.

Malhotra, H., Kumar, M., Chauhan, A.S., Dhiman, A., Chaudhary, S., Patidar, A., Jaiswal, P., Sharma, K., Sheokand, N., Raje, C.I. \& Raje, M. (2019). Moonlighting protein glyceraldehyde-3-phosphate dehydrogenase: a cellular rapidresponse molecule for maintenance of iron homeostasis in hypoxia. Cellular Physiology and Biochemistry 52: 517531.

Maurady, A., Zdanov, A., de Moissac, D., Beaudry, D. \& Sygusch, J. (2002). A conserved glutamate residue exhibits multifunctional catalytic roles in Dfructose-1,6-bisphosphate aldolases. Jourmal of Biological Chemistry 277: 9474-9483.

Merkulova, M., Hurtado-Lorenzo, A., Hosokawa, H., Zhuang, Z., Brown, D., Ausiello, D.A. \& Marshansky, V. (2011). Aldolase directly interacts with ARNO and modulates cell morphology and acidic vesicle distribution. American Journal of Physiology-Cell Physiology 300: C1442-1455.

Mulvenna, J., Sripa, B., Brindley, P.J., Gorman, J., Jones, M.K., Colgrave, M.L., Jones, A., Nawaratna, S., Laha, T., Suttiprapa, S., Smout, M.J. \& Loukas, A. (2010). The secreted and surface proteomes of the adult stage of the carcinogenic human liver fluke Opisthorchis viverrini. Proteomics 10: 1063-1078. 
Mundodi, V., Kucknoor, A.S. \& Alderete, J.F. (2008). Immunogenic and plasminogenbinding surface-associated alphaenolase of Trichomonas vaginalis. Infection and Immunity 76: 523-531.

Nagini, S., Sophia, J. \& Mishra, R. (2018). Glycogen synthase kinases: moonlighting proteins with theranostic potential in cancer. Seminars in Cancer Biology.

Pak, J.H., Moon, J.H., Hwang, S.J., Cho, S.H., Seo, S.B. \& Kim, T.S. (2009). Proteomic analysis of differentially expressed proteins in human cholangiocarcinoma cells treated with Clonorchis sinensis excretory-secretory products. Journal of Cellular Biochemistry 108: 1376-1388.

Perez-Sanchez, R., Ramajo-Hernandez, A., Ramajo-Martin, V. \& Oleaga, A. (2006). Proteomic analysis of the tegument and excretory-secretory products of adult Schistosoma bovis worms. Proteomics 6 Suppl 1: S226-236.

Plow, E.F., Herren, T., Redlitz, A., Miles, L.A. \& Hoover-Plow, J.L. (1995). The cell biology of the plasminogen system. The FASEB Journal 9: 939-945.

Ramajo-Hernandez, A., Perez-Sanchez, R., Ramajo-Martin, V. \& Oleaga, A. (2007). Schistosoma bovis: plasminogen binding in adults and the identification of plasminogen-binding proteins from the worm tegument. Experimental Parasitology 115: 83-91.

Rim, H.J. (1986). The current pathobiology and chemotherapy of clonorchiasis. The Korean Journal of Parasitology 24 Suppl: 1-141.

Rodriguez-Bolanos, M. \& Perez-Montfort, R. (2019). Medical and veterinary importance of the moonlighting functions of triosephosphate isomerase. Current Protein \& Peptide Science 20: 304-315.

Schindler, R., Weichselsdorfer, E., Wagner, O. \& Bereiter-Hahn, J. (2001). Aldolaselocalization in cultured cells: cell-type and substrate-specific regulation of cytoskeletal associations. Biochemistry and Cell Biology 79: 719-728.

Shams, F., Oldfield, N.J., Lai, S.K., Tunio, S.A., Wooldridge, K.G. \& Turner, D.P. (2016). Fructose-1,6-bisphosphate aldolase of Neisseria meningitidis binds human plasminogen via its C-terminal lysine residue. Microbiologyopen 5: 340-350.

Shams, F., Oldfield, N.J., Wooldridge, K.G. \& Turner, D.P. (2014). Fructose-1,6bisphosphate aldolase (FBA)-a conserved glycolytic enzyme with virulence functions in bacteria: "ill met by moonlight'. Biochemical Society Transactions 42: 1792-1795.

Sirover, M.A. (2018). Pleiotropic effects of moonlighting glyceraldehyde-3phosphate dehydrogenase (GAPDH) in cancer progression, invasiveness, and metastases. Cancer and Metastasis Reviews 37: 665-676.

Sripa, B., Kaewkes, S., Sithithaworn, P., Mairiang, E., Laha, T., Smout, M., Pairojkul, C., Bhudhisawasdi, V., Tesana, S., Thinkamrop, B., Bethony, J.M., Loukas, A. \& Brindley, P.J. (2007). Liver fluke induces cholangiocarcinoma. PLoS Medicine 4: e201.

Starnes, G.L., Coincon, M., Sygusch, J. \& Sibley, L.D. (2009). Aldolase is essential for energy production and bridging adhesin-actin cytoskeletal interactions during parasite invasion of host cells. Cell Host \& Microbe 5: 353-364.

Tochio, T., Tanaka, H., Nakata, S. \& Hosoya, H. (2010). Fructose-1,6-bisphosphate aldolase A is involved in HaCaT cell migration by inducing lamellipodia formation. Journal of Dermatological Science 58: 123-129.

Tunio, S.A., Oldfield, N.J., Berry, A., Ala'Aldeen, D.A., Wooldridge, K.G. \& Turner, D.P. (2010). The moonlighting protein fructose-1,6-bisphosphate aldolase of Neisseria meningitidis: surface localization and role in host cell adhesion. Molecular Microbiology 76: 605-615.

Wang, X., Chen, W., Hu, F., Deng, C., Zhou, C., Lv, X., Fan, Y., Men, J., Huang, Y., Sun, J., Hu, D., Chen, J., Yang, Y., Liang, C., Zheng, H., Hu, X., Xu, J., Wu, Z. \& Yu, X. (2011a). Clonorchis sinensis enolase: identification and biochemical characterization of a glycolytic enzyme from excretory/secretory products. Molecular and Biochemical Parasitology 177: 135142. 
Wang, X., Chen, W., Huang, Y., Sun, J., Men, J., Liu, H., Luo, F., Guo, L., Lv, X., Deng, C., Zhou, C., Fan, Y., Li, X., Huang, L., Hu, Y., Liang, C., Hu, X., Xu, J. \& Yu, X. (2011b). The draft genome of the carcinogenic human liver fluke Clonorchis sinensis. Genome Biology 12: R107.
Wilson, R.A., Wright, J.M., de Castro-Borges, W., Parker-Manuel, S.J., Dowle, A.A., Ashton, P.D., Young, N.D., Gasser, R.B. \& Spithill, T.W. (2011). Exploring the Fasciola hepatica tegument proteome. International Journal Parasitology 41: 1347-1359. 